\title{
Electron Microscopy of Nafion Membrane
}

\author{
Sergey Yakovlev ${ }^{1}$, Kenneth H. Downing ${ }^{2}$, Nitash P Balsara ${ }^{1,3}$ \\ 1. Material Science Division, Lawrence Berkeley National Laboratory, Berkeley, CA 94720 USA \\ 2. Life Science Division, Lawrence Berkeley National Laboratory, Berkeley, CA 94720 USA \\ 3. Chemical and Biomolecular Engineering, University of California, Berkeley, CA 94710 USA
}

Polyelectrolyte membranes have considerable importance for d evelopment of energy-related technologies, most notably fuel cells. The majority of current generation fuel cell devices use Nafion as the polyelectrolyte membrane. While this material has been intensively studied for the last two decades, its nanostructure still has not been decisively resolved [1]. Most of the Nafion investigations rely on the results of SAXS experiments, which show a wide peak at several nanometers that has been related to the clustering of ionic groups in the material. Several contradicting models of Nafion structure have been suggested to explain this peak. All of these models describe the experimental data reasonably well and none of them can be selected over the others. Thus the real nanostructure of the material remains a subject of cont roversy. To $r$ esolve this uncertainty additional constraints have to $b$ e applied to the nanostructure models. While such constraints can be obtained by electron microscopy, the success of microscopy investigation so far has been moderate. Difficulties in electron microscopy study of Nafion nanostructure are related to its high electron beam sensitivity and relatively low contrast between the hydrophilic and hydrophobic phases [2]. In most of the electron microscopy studies, Nafion has been doped with heavy atoms to improve the contrast in the images. Doping, however, is well known to affect the material structure. In those few investigation that addressed the structure of unmodified acidic Nafion, the effects of electron irradiation-induced damage have not been discussed. Because Nafion is well known to be one of the most radiation sensitive polymeric materials, beam damage may significantly affect the results of the microscopy investigations.

Consistent with previous experiments, we found Nafion to be very sensitive to i rradiation. We also found that the effect of irradiation strongly depends on the dose rate. High dose rates caused severe mass loss and flow of material from irradiated areas that rapidly formed holes in the film. Irradiation with dose rates below $100 \mathrm{e} / \mathrm{nm}^{2}$ s typically did not produces visible changes in morphology. Extended irradiation with such doses stabilized the material and reduced its sensitivity to further irradiation. After the low dose rate irradiation the film was capable of withstanding high dose rates, which indicated that the chemical structure of the material had been somewhat modified. Images obtained under the low dose rate conditions (Figure 1) can clearly dismiss some of the Nafion nanostructure models. No aligned parallel channels or bundles of channels can be seen. Instead the morphology appears as disconnected (at the resolution of the experiment) clusters randomly scattered over the sample.

Interpretation of TEM images of Nafion is not straightforward. Ionic domains contain ether side chains ending with sulfuric acid groups. Sulfur atoms scatter the electron beam more strongly than the other components of Nafion, which may cause the clusters to appear dark in bright field images. On the other hand the density of the ether-rich region is less than the density of the polytetrafluoroethylene backbone region, which is crystalline and densely packed. The high density of the crystalline phase could make it look darker in bright field images than the ionic phase. We applied selective electron beam etching to preferentially remove the polytetrafluoroethylene and locate the $\mathrm{m}$ ore irradiation-stable hydrophilic phase. To facilitate the etching we studied the sample in STEM, where the focused, intense electron 
beam removes material from beam-sensitive parts of the sample. Figure 2 shows a HAADF image that clearly reveals the bright clusters distributed in a more uniform dark background.

Our investigation shows that morphology models suggesting the existence of continuous channels through the material are not supported by electron microscopy. Instead the hydrophilic domains appear isolated at the resolution of our experiments.

[1] Mauritz, K. and R. Moore, Chem Rev, 2004. 104.

[2] Yakovlev, S. and K.H. Downing, Physical Chemistry Chemical Physics, 2013, 15.

[3] This work was supported by Director, Office of Science, Office of Basic Energy Sciences, Materials Sciences and Engineering Division, of the U.S. Department of Energy; Grant Number: DE-AC02$05 \mathrm{CH} 11231$.

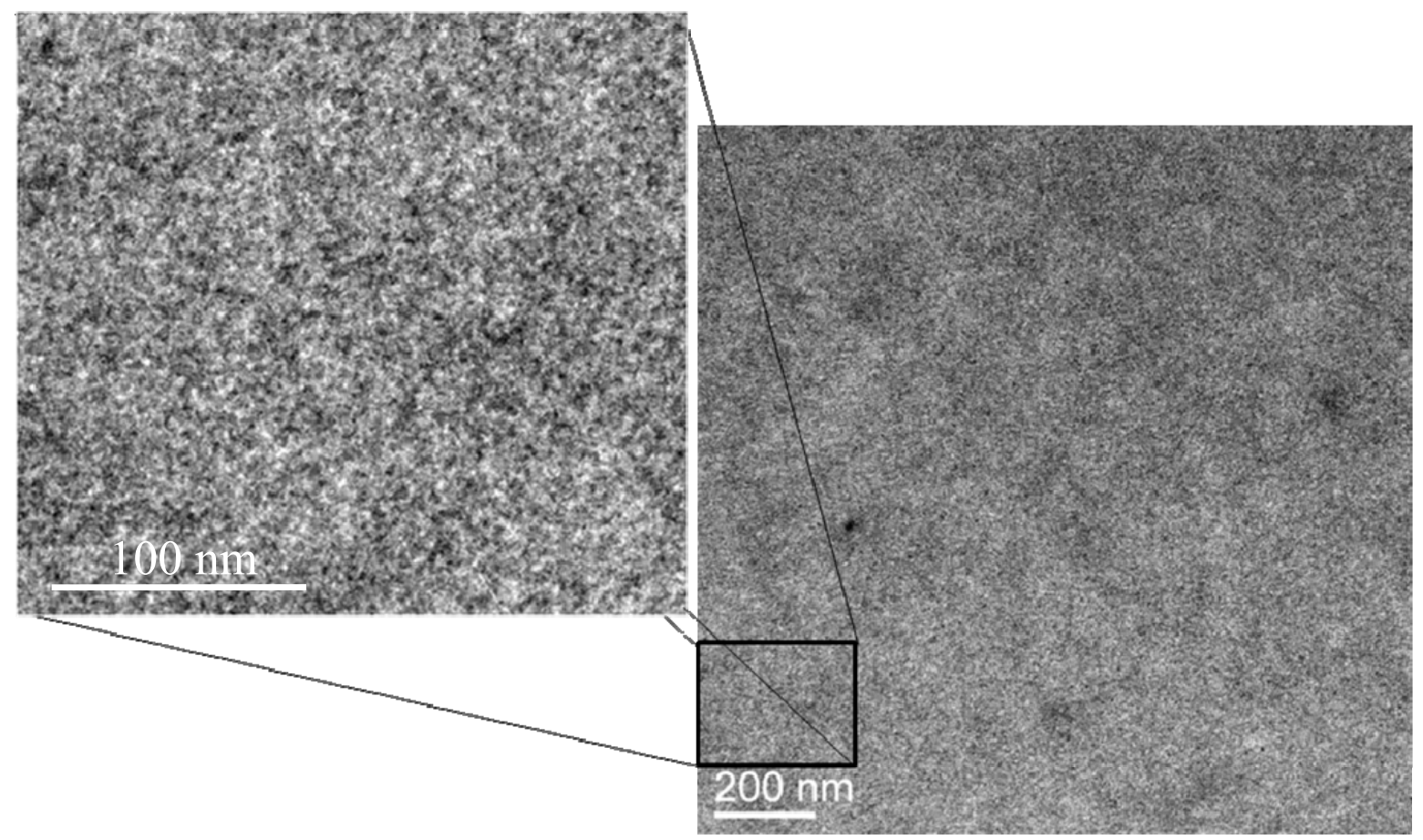

Figure 1. Low dose Bright field image of solvent-cast Nafion membrane

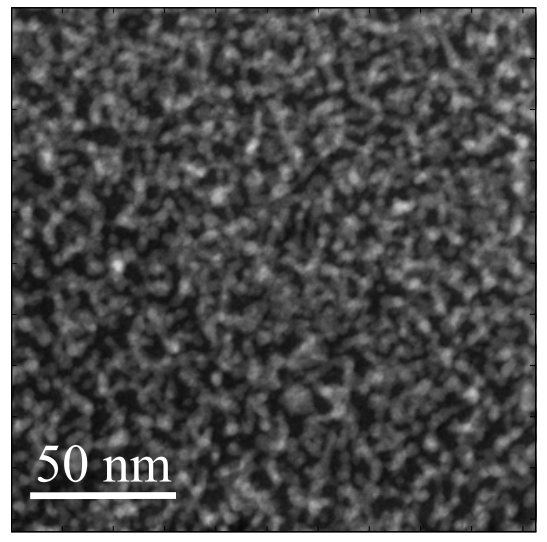

Figure 2. HAADF image of Nafion membrane after selective etching of hydrophilic phase with electron beam. 